\title{
A Case of Recurrent Atypical Anti-Glomerular Basement Membrane Nephritis Suspicion after Renal Transplantation
}

\author{
Shinsuke Isobe Toshihide Tomosugi Kenta Futamura Manabu Okada \\ Takahisa Hiramitsu Makoto Tsujita Shunji Narumi Norihiko Goto \\ Asami Takeda Yoshihiko Watarai \\ Department of Kidney Disease Center, Nagoya Daini Red Cross Hospital, Nagoya, Japan
}

\section{Keywords}

Anti-GBM antibody disease - Atypical anti-GBM nephritis .

De novo glomerulonephritis · Focal segmental

glomerulosclerosis $\cdot$ Kidney transplantation

\begin{abstract}
Atypical anti-glomerular basement membrane (GBM) nephritis is a rare variant of the classical anti-GBM antibody disease. Patients present with an undetectable anti-GBM antibody but show linear glomerular basement membrane staining for immunoglobulin. We present a 69-year-old man who underwent a living-donor kidney transplant. The aetiology of the renal failure was a focal segmental glomerulonephritis-like lesion resistant to immunosuppressive therapy. A renal graft biopsy revealed diffuse endocapillary hypercellularity, and mild mesangiolysis with linear GBM staining for lgG. The patient was diagnosed with atypical anti-GBM nephritis since the patient tested negative for circulating antiGBM antibodies. Treatment involved intravenous methylprednisolone, plasma exchange, and rituximab administration. Protocol graft biopsy performed 1 year after the renal transplant showed a focal segmental glomerulonephritis-
\end{abstract}

like lesion possibly progressing from endocapillary hypercellularity and mesangiolysis. These findings were similar to his native kidney biopsy findings. Although classical recurrent anti-GBM nephritis is rare when a renal transplant is performed after decreased disease activity, this case was considered as a case of recurrent atypical anti-GBM nephritis after renal transplant.

(c) 2020 S. Karger AG, Basel

\section{Introduction}

Anti-glomerular basement membrane (GBM) antibody disease is one of the most common types of fulminant nephritis, characterized by a rapidly progressive glomerulonephritis due to necrotizing vasculitis with linear GBM staining for IgG on immunofluorescence (IF). Approximately $40-60 \%$ of patients present with alveolar haemorrhage $[1,2]$. These morbidities are caused by the anti-GBM antibody, which is a circulating autoantibody directed against the $\mathrm{N}$-terminal of the non-collagen 1 domain of the alpha- 3 chain ( $\alpha 3 \mathrm{NC} 1)$ of type IV collagen. However, about $10 \%$ of patients with anti-GBM antibody karger@karger.com

www.karger.com/nef

Karger ${ }^{\prime}=$
(C) 2020 S. Karger AG, Basel

(n)
Shinsuke Isobe

Internal Medicine 1, Hamamatsu University School of Medicine

1-20-1 Handayama, Higashi-ku

Hamamatsu 431-3192 (Japan)

isobe58@hama-med.ac.jp 

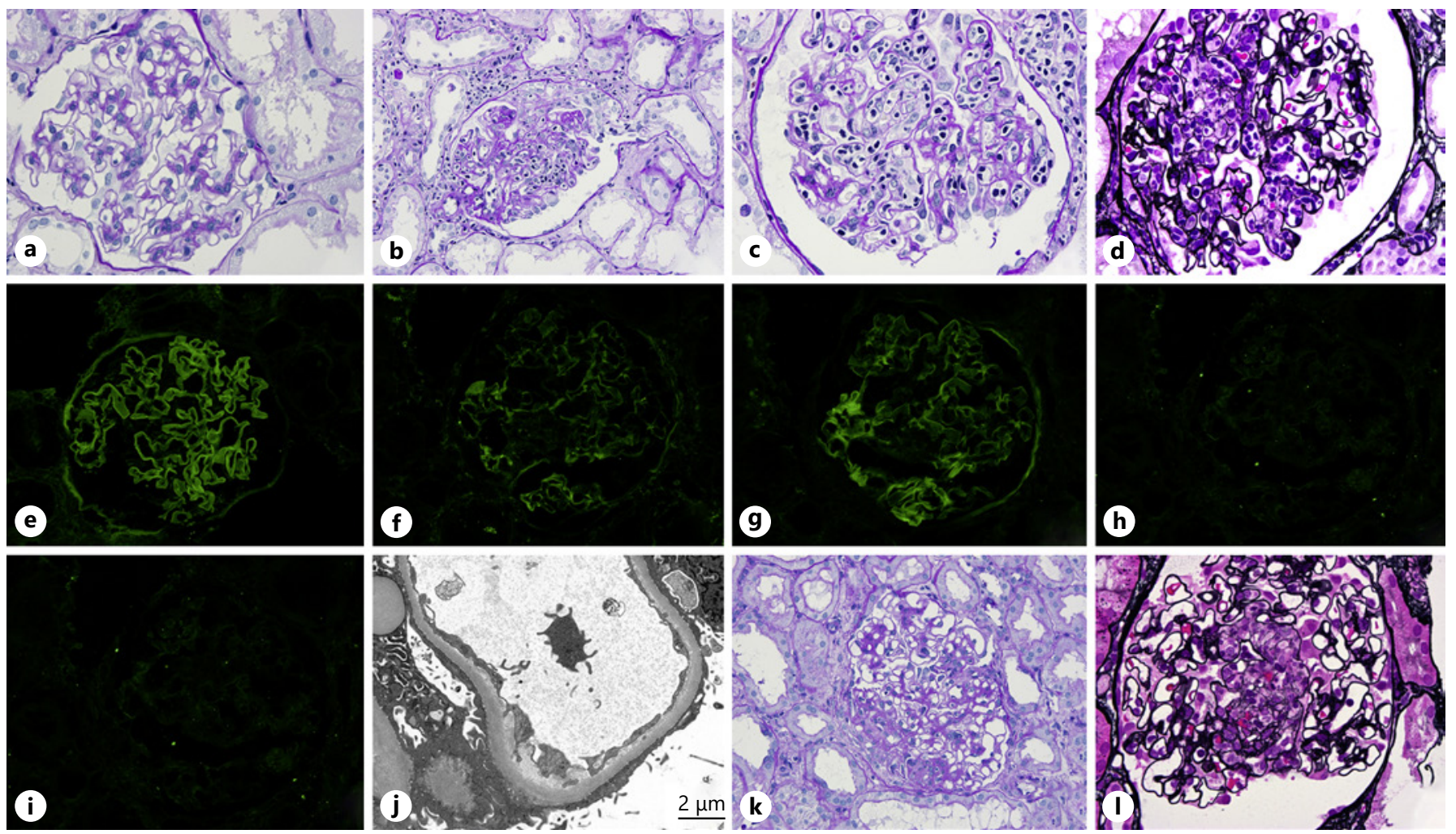

Fig. 1. Light microscopic findings, IF findings of IgG and IgG subclass and findings of electron microscopy. a Protocol biopsy $1 \mathrm{~h}$ after renal transplantation. Although mild mesangial proliferation existed, there were no findings of intracapillary nor extracapillary proliferation (PAS stain: original magnification, $\times 400$ ); Episode biopsy 3 months after surgery (b-d), Extracapillary proliferation (PAS: original magnification, $\times 200)(\mathbf{b})$, Diffuse endocapillary hypercellularity and mesangiolysis (PAS; original magnification, $\times 400$ (c), PAM: original magnification, $\times 400(\mathbf{d}))(\mathbf{c}$, d); Linear GBM staining for IgG. IF finding of IgG (original magnification, $\times 400)(\mathbf{e})$; Liner GBM staining for IgG1 and $2(\operatorname{IgG} 1<2)$ but negative for IgG3 and 4 (IgG1 (f), IgG2 (g), IgG3 (h), IgG4 (i); original magnification, $\times 400)(\mathbf{f}-\mathbf{i})$; Findings of electron microscopy at 3 months after surgery (j). Diffuse foot process effacement and subendothelial space widening are observed. Electron-dense deposits are absent in the subendothelial, intramembranous, subendothelial, and mesangial regions (original magnification, $\times 1,400$ ). $\mathbf{k}$, I Protocol biopsy performed at 1 year postoperatively: Segmental sclerosis with intracapillary hypercellularity was observed in some glomeruli (PAS original magnification, $\times 200)(\mathbf{k})$, Mesangiolysis occurred in focal glomeruli (PAM original magnification, $\times 400$ ) (I). IF, immunofluorescence; PAS, periodic acid-Schiff; PAM, periodic acid-methenamine; GBM, glomerular basement membrane.

\section{Case Report}

A 64-year-old Japanese man was diagnosed with nephrotic syndrome with microscopic haematuria, after he developed proteinuria and haematuria at 28 years of age. His renal function deteriorated (serum $\mathrm{Cr}=1.3 \mathrm{mg} / \mathrm{dL}$ ). A percutaneous renal biopsy was performed at 61 years of age, and a focal segmental glomerulosclerosis (FSGS)-like lesion with endocapillary proliferation was diagnosed. Although the patient received prednisolone, mizoribine, and LDL-apheresis, his renal function gradually worsened. $\mathrm{He}$ started maintenance haemodialysis at 66 years of age, and 3 years later, at 69 years of age, he was admitted to our hospital for a renal transplant.

An ABO-compatible living-related renal transplant was successfully completed. He received the kidney from his brother. $\mathrm{Hu}$ man leukocyte antigen alleles were mismatched at 5 , the standard
Isobe et al. 
Fig. 2. a, b Native kidney biopsy findings at 61 years old. Focal segmental sclerosis has diagnosed, though intracapillary proliferation and podocyte hypertrophy have also existed. (PAS original magnification, $\times 200$ (a), PAM, original magnification, $\times 1,000$ (b)).
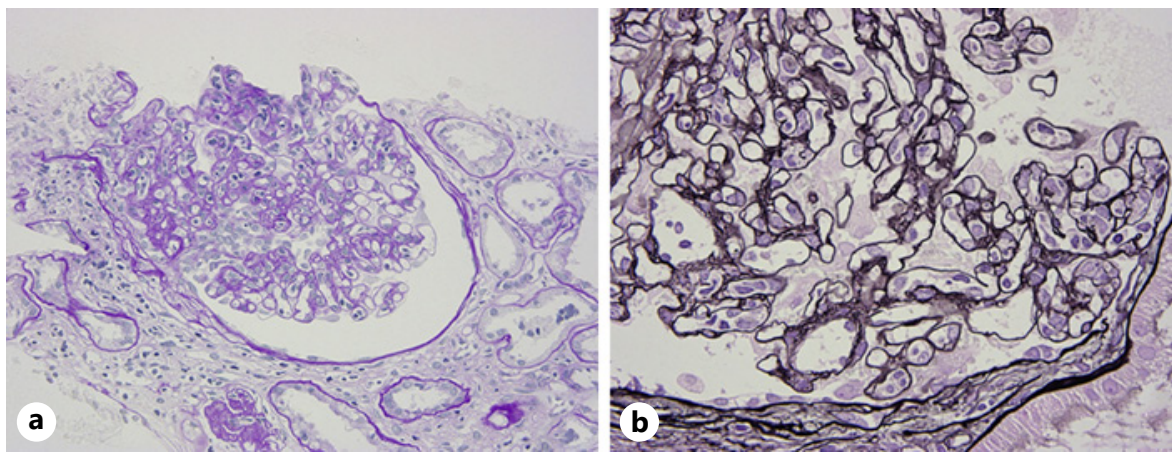

Fig. 3. Clinical course after renal transplantation. After diagnosis of atypical antiGBM nephritis, the patient received 500 mg methylprednisolone intravenously for 3 days, plasma exchange 3 times, and 200 $\mathrm{mg}$ of rituximab. His proteinuria did not decrease but renal function was maintained. KTX, kidney transplantation; GBX, graft biopsy, CMV, cytomegalovirus infection; CyA, cyclosporine, MMF, mycophenolate mofetil; PSL, prednisolone; MP, methylprednisolone; PEX, plasma exchange.

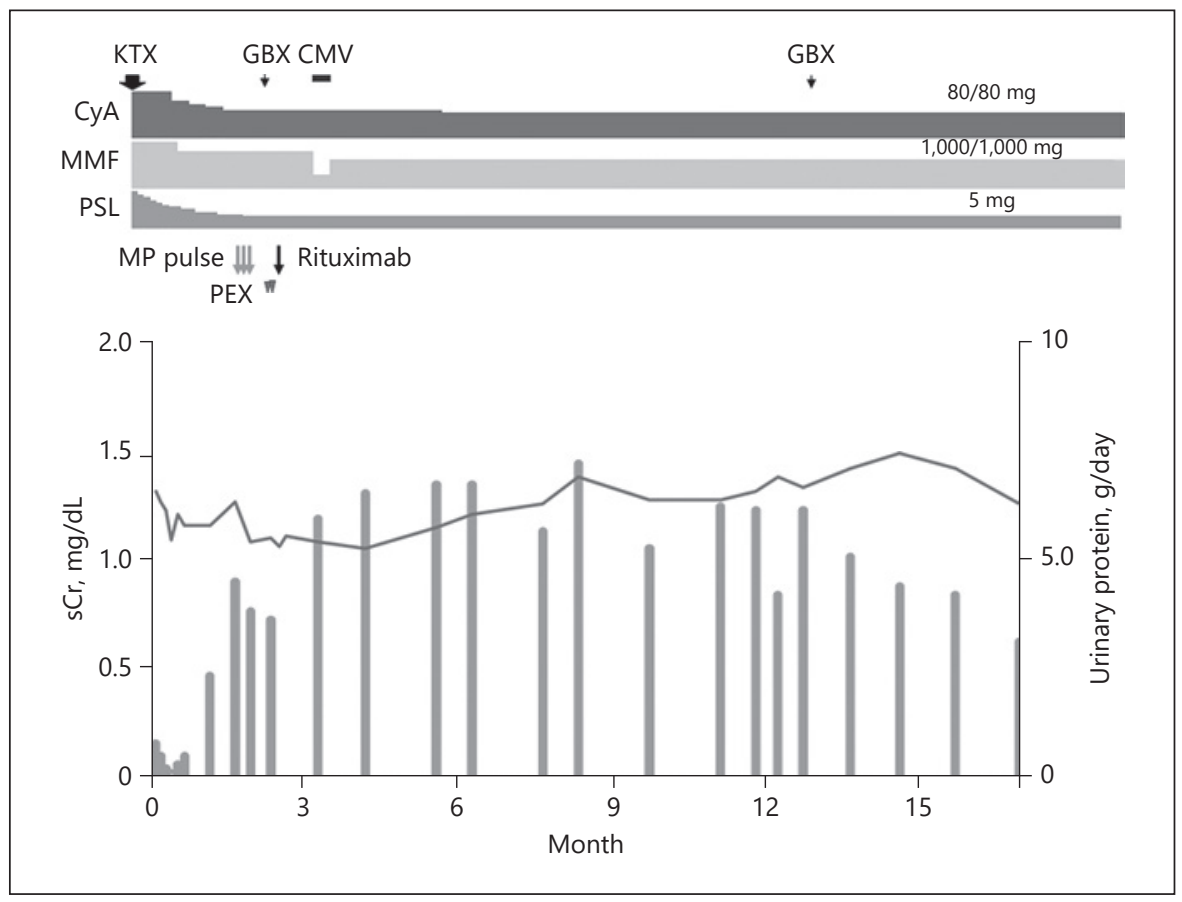

complement-dependent cytotoxicity and flow cytometry crossmatch tests were negative, and donor-specific antibodies were not detected. Immunosuppressive therapy included basiliximab, prednisolone, cyclosporine, and mycophenolate mofetil. The 1 -h biopsy revealed mild mesangial proliferation, and an IF analysis showed no obvious immunoglobulin depositions (Fig. 1a). The serum $\mathrm{Cr}$ level improved to $1.11 \mathrm{mg} / \mathrm{dL}$ on postoperative day 14 . Nonetheless, haematuria and proteinuria at $0.5 \mathrm{~g} /$ day continued. At postoperative day 93, a graft biopsy was conducted due to worsening of proteinuria with microscopic haematuria (30-49/high power field) (Fig. 3). The renal histological examination showed diffuse endocapillary hypercellularity, mesangiolysis, and extracapillary proliferation (Fig. 1b-d). The IF studies showed a linear GBM stain for IgG (IgG1 and 2 but not IgG3 and 4) but was negative for other immunoglobulins, complement components, and C4d (Fig. 1e-i). There was no immunoglobulin stain in other areas such as mesangial lesion, tubular basement membrane, or peritubular capillary. Electron microscopy findings indicated diffuse foot process effacement and subendothelial space widening (Fig. 1j). Moreover, not only serum anti-GBM antibody, myeloperoxidase and proteinase 3 antineutrophil cytoplasmic antibody but also de novo donor-specific antigen was negative. Therefore, an atypical anti-GBM nephritis was diagnosed. We reanalysed the patient for native kidney pathologies. Although IF studies were not performed since there were no remaining specimens, the FSGS like lesions with endocapillary hypercellularity were theorized to be caused by thrombotic microangiopathy and mesangiolysis (Fig. 2a, b).

Treatment involved intravenous administration of $500 \mathrm{mg}$ of methylprednisolone for 3 days, plasma exchange 3 times a day, and administration of $200 \mathrm{mg}$ of rituximab. Though the renal function did not worsen, proteinuria persisted at a nephrotic range (Fig. 3). The protocol 1-year graft biopsy after renal transplantation showed the FSGS possibly progressing from endocapillary hypercellularity and mesangiolysis (Fig. 1k, l). There was no clinical evidence of lung involvement during the follow-up period. 


\section{Discussion}

An atypical anti-GBM nephritis is a rare variant of the classical anti-GBM antibody disease. Nasr et al. [3] have reported clinical and pathological characteristics of 20 atypical anti-GBM nephritis patients. Half of them had endocapillary hypercellularity, and $10 \%$ of patients were diagnosed with FSGS on light microscopy. Out of these patients, 50\% revealed monoclonal immunoglobulin deposition, and 50, 29, and $42 \%$ of the patients showed linear GBM staining for IgG1, IgG2, and IgG4 dominant or co-dominant, respectively. In patients with anti-GBM antibody disease, autoantibodies to the $\alpha 3 \mathrm{NC} 1$ monomer were present. The commercially available ELISA kit detects circulating IgG1 anti-GBM antibodies, although other IgG subclasses or other immunoglobulins such as IgA and IgM can also cause a morbid state. The anti-GBM disease with a predominance of IgG4 is known as a mild renal insufficiency form, possibly due to the low affinity of IgG4 to bind complement [5]. Although the renal histological findings were compatible with atypical antiGBM nephritis, IgG1 and 2 but not IgG4, IgA, or IgM staining was dominant in our case. Although we could not completely rule out non-specific absorption of IgG, we could rule out other morbidities showing linear GBM staining for IgG such as diabetes mellites, monoclonal IgG deposition, and classical anti-GBM antibody disease. The other possibilities for these negative results might be the underpowered standard ELISA kit [6], different antigens, or epitopes reacting with $\alpha 3 \mathrm{NC} 1$ [7]. There was no direct evidence to diagnose the case as an atypical antiGBM nephritis in native kidney biopsy findings because we could not analyse the immunoglobulin deposition as no specimen remained. However, this seemed to be a case of recurrent atypical anti-GBM nephritis after renal transplant for the following reasons. Firstly, due to the diverse nature of microscopic findings, a diagnosis of an atypical anti-GBM nephritis is challenging $[1,3]$. Therefore, the case might have been incorrectly diagnosed as FSGS. Secondly, a de novo anti-GBM nephritis is unlikely to develop early after a renal transplant if the underlying renal disease is different. Thirdly, the FSGS with intracapillary hypercellularity diagnosed 1 year after renal transplant had similar findings with the native kidney biopsy. The FSGS is a histological diagnosis but has several different aetiologies $[8,9]$. Although primary FSGS presumably occurred and the circulating factors recurred immediately after the kidney transplant, secondary FSGS caused hyperfiltration due to nephron damages and did not recur if nephron damage had occurred.
In classical anti-GBM antibody disease patients, about $50 \%$ of histological recurrence of linear GBM staining for IgG occurred after a renal transplant [10]. However, most patients remained clinically asymptomatic if renal transplant was conducted when the disease activity was diminished [1]. In this case, we may have performed a renal transplant while the disease was active, and that might be one of the reasons for a recurrent atypical anti-GBM nephritis after the renal transplant although we did not test for circulating autoantibodies.

Because it is a rare disease, there are no published papers about the appropriate treatment for recurrent atypical anti-GBM nephritis after renal transplant [11-13]. The conventional immunosuppression treatment for renal transplant resulted in disease relapse, so a more aggressive treatment consisting of intravenous methylprednisolone pulse, plasma exchange, and rituximab was undertaken to diminish disease activity. However, there is a need for other methods to treat this disease.

In conclusion, we reported a case of suspicious recurrent atypical anti-GBM nephritis after renal transplant, which is a rare variant of the classical anti-GBM antibody disease. Although there were no high-quality evidences, the atypical anti-GBM nephritis slowly worsened and resisted conventional immunosuppressive treatment.

\section{Statement of Ethics}

Written informed consent was obtained from the patient.

\section{Conflict of Interest Statement}

The authors have no conflict of interest to declare.

\section{Funding Sources}

The authors did not receive any funding.

References

1 McAdoo SP, Pusey CD. Anti-glomerular basement membrane disease. Clin J Am Soc Nephrol. 2017;12(7):1162-72.

2 Lazor R, Bigay-Gamé L, Cottin V, Cadranel J, Decaux O, Fellrath J-M, et al. Alveolar haemorrhage in anti-basement membrane antibody disease: a series of 28 cases. Medicine. 2007;86:181-93.

3 Nasr SH, Collins AB, Alexander MP, Schraith DF, Herrera Hernandez L, Fidler ME, et al. The clinicopathologic characteristics and outcome of atypical anti-glomerular basement membrane nephritis. Kidney Int. 2016;89(4):897-908.
Isobe et al. 
4 Tang W, McDonald SP, Hawley CM, Badve SV, Boudville NC, Brown FG, et al. Anti-glomerular basement membrane antibody disease is an uncommon cause of end-stage renal disease. Kidney Int. 2013;83(3):503-10.

5 Ohlsson S, Herlitz H, Lundberg S, Selga D, Mölne J, Wieslander J, et al. Circulating antiglomerular basement membrane antibodies with predominance of subclass IgG4 and false-negative immunoassay test results in anti-glomerular basement membrane disease. Am J Kidney Dis. 2014;63(2):289-93.

6 Salama AD, Dougan T, Levy JB, Cook HT, Morgan SH, Naudeer S, et al. Goodpasture's disease in the absence of circulating anti-glomerular basement membrane antibodies as detected by standard techniques. Am J Kidney Dis. 2002;39(6):1162-7.
7 Hellmark T, Segelmark M, Wieslander J. Anti-GBM antibodies in Goodpasture syndrome: anatomy of an epitope. Nephrol Dial Transplant. 1997;12:646-8.

8 Rosenberg AZ, Kopp JB. Focal segmental glomerulosclerosis. Clin J Am Soc Nephrol. 2017;12(3):502-17.

9 Fogo AB. Causes and pathogenesis of focal segmental glomerulosclerosis. Nat Rev Nephrol. 2015;11(2):76-87.

10 Kotanko P, Pusey CD, Levy JB. Recurrent glomerulonephritis following renal transplantation. Transplantation. 1997;63(8):1045-52.
11 Sauter M, Schmid H, Anders HJ, Heller F, Weiss M, Sitter T. Loss of a renal graft due to recurrence of anti-GBM disease despite rituximab therapy. Clin Transplant. 2009;23(1): 132-6.

12 Khandelwal M, McCormick BB, Lajoie G, Sweet J, Cole E, Cattran DC. Recurrence of anti-GBM disease 8 years after renal transplantation. Nephrol Dial Transplant. 2004; 19(2):491-4.

13 Borza DB, Chedid MF, Colon S, Lager DJ, Leung N, Fervenza FC. Recurrent Goodpasture's disease secondary to a monoclonal IgA1-kappa antibody autoreactive with the alpha1/alpha2 chains of type IV collagen. Am J Kidney Dis. 2005;45(2):397-406. 